\title{
Investigating an outbreak of staphylococcal food poisoning among travellers across two Australian states
}

\author{
Stephanie Fletcher, ${ }^{a}$ Leng Boonwaat, ${ }^{a}$ Terry Moore, ${ }^{b}$ Ruchir Chavada ${ }^{c}$ and Stephen Conaty ${ }^{a}$ \\ Correspondence to Stephanie Fletcher (e-mail: stephanie.fletcher@sswahs.nsw.gov.au).
}

Introduction: Staphylococcus aureus is a common cause of staphylococcal food poisoning in Australia with several outbreaks associated with foods prepared by commercial caterers. Laboratory testing on cases of gastrointestinal illness caused by enterotoxin-producing S. aureus is not routinely done as this condition is self-limiting. Hence outbreaks of such illness may go undetected.

Methods: A retrospective cohort study was conducted among a group of tourists who were hospitalized in Sydney shortly after flying from Queensland. The group had consumed food prepared by a restaurant on the Gold Coast before transit. Laboratory analyses on stool specimens were conducted in Sydney. An environmental assessment of the restaurant in the Gold Coast was conducted, and environmental specimens were assessed for contamination.

Results: Epidemiological investigations linked the outbreak to a restaurant in the Gold Coast where the suspected food was produced. Stool samples from two of the hospitalized cases were confirmed to have enterotoxin-producing S. aureus, and several environmental samples were found to be contaminated with $S$. aureus as well. Investigations suggested that absence of hand washing and other unhygienic food handling at the implicated restaurant was the likely cause of this outbreak.

Conclusion: Food poisoning due to toxin-mediated S. aureus is frequently undetected and underreported. Public health units should consider toxin-producing pathogens such as $S$. aureus when investigating outbreaks where vomiting is the predominant symptom and occurs rapidly after consuming food.

E nterotoxin-producing Staphylococcus aureus causes toxin-mediated food poisoning with an estimated 1300 cases reported annually in Australia. ${ }^{1}$ Intoxication or staphylococcal food poisoning (SFP) occurs following ingestion of food products contaminated with heat-resistant $S$. aureus enterotoxins. ${ }^{2}$ Food handlers carrying enterotoxin-producing $S$. aureus in their noses or on their hands are the main source of food contamination via direct contact or through respiratory secretions. Foods high in starch and protein are believed to favour staphylococcal enterotoxin (SE) production. Staphylococcal food poisoning (SFP) symptoms generally have a rapid onset, appearing within three hours after ingestion (range: 30 minutes to 6 hours). Common symptoms include nausea, vomiting, abdominal cramps and diarrhoea. Fever is absent. Recovery usually occurs between 1 and 3 days.

On 28 October 2014, the local Public Health Unit in South Western Sydney Local Health District was notified by a hospital emergency department (ED) of an outbreak.
A group of Japanese tourists travelling from Brisbane on an organized tour experienced sudden onset of vomiting and diarrhoea shortly after landing in Sydney. Twelve of the 27 passengers experienced multiple episodes of vomiting with onsets occurring progressively within 10-30 minutes of each other. All cases were assessed in the ED, and four were hospitalized overnight for observation.

\section{METHODS}

\section{Case finding}

A case was defined as any member of the tour who travelled from the Gold Coast and experienced vomiting and/or diarrhoea between $11: 00$ and $16: 00$ on 28 October 2014. A cohort investigation was conducted to identify risk factors. A line listing including demographic details and clinical and food histories was prepared by Public Health Unit and/or ED staff with the assistance of an interpreter. Based on the three-day tour schedule,

\footnotetext{
Public Health Unit, South Western Sydney Local Health District, New South Wales, Australia

Environmental Health Services, Public Health Unit, Gold Coast, Queensland, Australia.

Sydney South West Pathology Service, Liverpool Hospital, New South Wales, Australia.

Submitted: 9 February 2015; Published: 4 May 2015

doi: 10.5365/wpsar.2015.6.1.011
} 
Figure 1. Number of cases by symptom onset, New South Wales, Australia, 2014

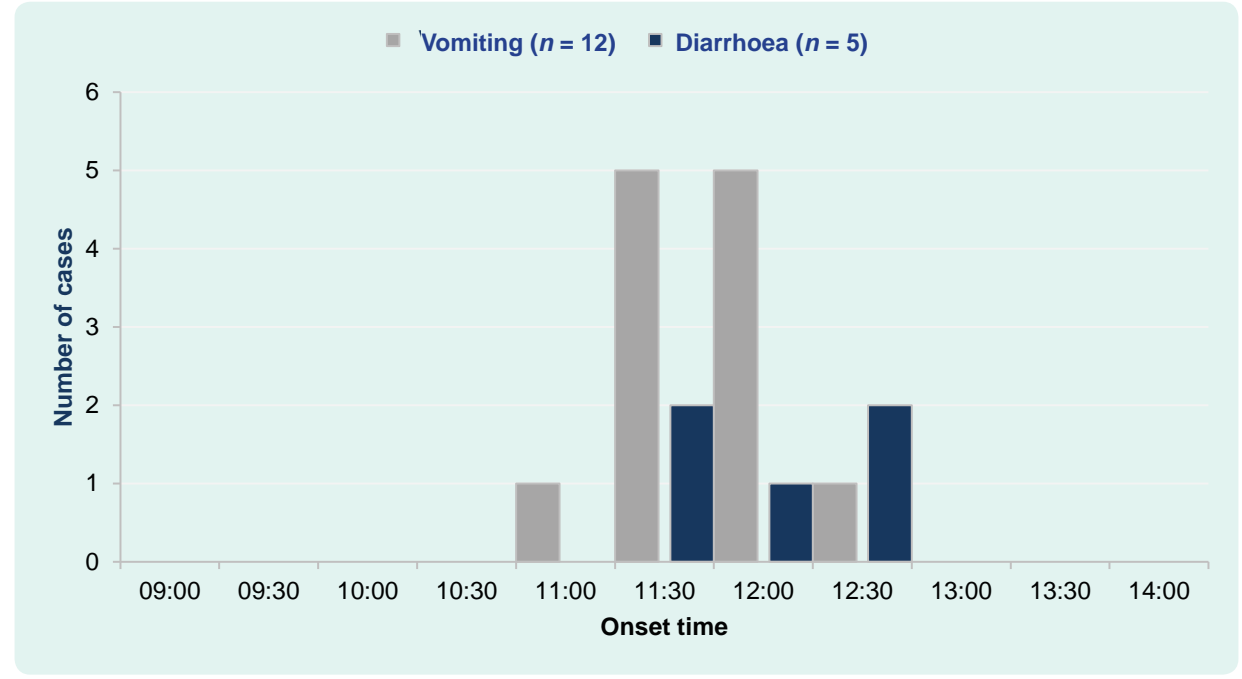

the group's team leader believed that it was unlikely that anyone had consumed food outside of scheduled meals. Food-specific attack rates and risk ratios were calculated for each food item. Analysis was conducted using Microsoft Excel.

\section{Laboratory investigations}

Stool samples were collected from three hospitalized patients and tested using the routine direct molecular (triplex) test for detection of Salmonella, Shigella and Campylobacter..$^{3-5}$ Norovirus antigen testing (using enzyme immunoassays) and cultures for Staphylococcus were also performed on the stool specimens using Columbia colistin nalidixic agar with sheep blood (Thermo Fischer Scientific, Scoresby, Victoria, Australia). A multiplex polymerase chain reaction (PCR) for staphylococcal enterotoxin $A$ to $E$ based on published primers was then performed on the $S$. aureus isolates. ${ }^{6}$ This multiplex PCR has only been validated on cultured bacterial isolates rather than direct detection from stool specimen. Blood cultures were done on all admitted patients for the detection of sepsis.

\section{Environmental investigations}

Environmental investigations were conducted by the Environmental Health Services at Gold Coast Public Health Unit in Queensland and also by the City of Gold Coast at the restaurant in question on 30 October 2014, two days after the food was consumed. A range of food samples and environmental swabs were collected including samples of boiled rice remaining from the tour group's breakfast. Food samples were assessed in accordance with the Food Standards Australia New Zealand Ready to Eat Guidelines. ${ }^{7}$ Environmental swabs were collected and cultured for the presence of Escherichia coli, S. aureus, Bacillus cereus and Salmonella spp.

\section{RESULTS}

\section{Clinical and epidemiological results}

Twelve (44\%) members of the group met the case definition; 10 were females. All 12 (100\%) cases experienced vomiting and five (42\%) experienced both diarrhoea and vomiting. No case of fever or headache was reported. The onset of illness was acute with all cases having onsets within two hours of the index case (Figure 1). Incubation periods ranged from 3 to 4.5 hours (mean 3.5 hours). The first case had an onset approximately 30 minutes after arrival at the airport in Sydney, characterized by profuse projectile vomiting. The second case's onset occurred approximately 15 minutes later followed by a period of concurrent vomiting and diarrhoea. Four elderly patients who had dehydration were admitted to hospital for intravenous hydration. Symptoms lasted for approximately 12 hours, and all cases were asymptomatic by the next morning. No clinical history was available for four group members.

Food histories were available for 23 of the 27 persons. All meals were catered and provided as part 
Table 1. Attack rates, risk differences and relative risks for food items consumed by cohort, New South Wales, Australia, 2014

\begin{tabular}{|c|c|c|c|c|c|c|c|c|c|c|}
\hline \multirow{3}{*}{ Food } & \multicolumn{6}{|c|}{ Number of persons who } & \multirow{3}{*}{$\begin{array}{l}\text { Difference } \\
\text { (\%) }\end{array}$} & \multirow{3}{*}{$\begin{array}{l}\text { Risk } \\
\text { ratio }\end{array}$} & \multirow{3}{*}{$\begin{array}{l}\text { Lower } \\
95 \% \mathrm{Cl}\end{array}$} & \multirow{3}{*}{$\begin{array}{l}\text { Upper } \\
95 \% \mathrm{Cl}\end{array}$} \\
\hline & \multicolumn{3}{|c|}{ Ate specified food } & \multicolumn{3}{|c|}{ Did not eat specified food } & & & & \\
\hline & Sick & Total & \% sick & Sick & Total & $\%$ sick & & & & \\
\hline Sushi/rice ball & 12 & 22 & 54.5 & 0 & 1 & 0.0 & 54.5 & 0 & - & - \\
\hline Fried chicken & 11 & 21 & 52.4 & 1 & 2 & 50.0 & 2.4 & 1.0 & 0.2 & 4.4 \\
\hline Pickled grilled salmon & 12 & 22 & 54.5 & 0 & 1 & 0.0 & 54.5 & 0 & - & - \\
\hline Bottled water & 3 & 7 & 42.8 & 9 & 16 & 56.2 & -13.4 & 0.8 & 0.3 & 1.9 \\
\hline Yogurt & 11 & 21 & 52.4 & 1 & 2 & 50.0 & 2.38 & 1.0 & 0.2 & 4.4 \\
\hline Dried fruit & 6 & 10 & 60.0 & 6 & 13 & 46.1 & 13.8 & 1.3 & 0.6 & 2.8 \\
\hline Muesli & 4 & 8 & 50.0 & 8 & 15 & 53.3 & -3.3 & 0.9 & 0.4 & 2.2 \\
\hline Coffee with milk & 9 & 14 & 64.3 & 3 & 9 & 33.3 & 30.9 & 1.9 & 0.7 & 5.3 \\
\hline Tea with milk & 2 & 7 & 28.6 & 10 & 16 & 62.5 & -33.9 & 0.5 & 0.1 & 1.5 \\
\hline Water - plain & 4 & 9 & 44.4 & 8 & 14 & 57.1 & -12.7 & 0.8 & 0.3 & 1.8 \\
\hline Orange juice & 1 & 1 & 100.0 & 11 & 22 & 50.0 & 50.0 & 2.0 & 1.3 & 3.0 \\
\hline
\end{tabular}

$\mathrm{Cl}$, confidence interval.

of the tour. On 28 October, on departure from the Gold Coast, the group was provided with packaged meals that were consumed at the airport before the 08:25 flight to Sydney. The meal included sushi (also described as rice ball) with pickled grilled salmon, fried chicken and bottled water. All 27 persons consumed the packaged food at the airport. During the flight at approximately 10:00, group members had a light breakfast which included yogurt, drief fruit, muesli, assorted juices and tea/coffee. All but one of the 23 persons consumed the breakfast items (Table 1).

Slightly more cases consumed both sushi/rice ball, chicken and pickled salmon with attack rates and rate differences of $55 \%$ for sushi and pickled grilled salmon and $52 \%$ for fried chicken. The risk ratios for the sushi and pickled grilled salmon were undefined (infinite) because all ill people ate these food items (no one who did not eat these items fell ill).

\section{Microbiological and environmental results}

All stool samples obtained from cases were negative for norovirus and all other microorganisms. S. aureus was detected in two of three specimens. Enterotoxin PCR detected presence of $S$. aureus enterotoxin $A$ and $D$ in both specimens. Blood cultures were negative.
The boiled rice was of unsatisfactory bacteriological quality based on a high standard plate count, and Salmonella species were detected at potentially hazardous levels. Seven of 10 swabs were positive for an enteric pathogen. S. aureus was detected in swabs taken from the sink, refrigerator door and dish cloth at the restaurant. $B$. cereus was found on the bench top, chopping board and refrigerator door.

Environmental investigation revealed inadequate hand-washing facilities for food handlers at the premise; food handlers only used hand sanitizer to cleanse their hands. None of the food handlers had symptoms of skin/ soft tissue infection or any open wounds. Potentially hazardous food was transported without adequate temperature control. Enforcement action was taken at the restaurant as per current Public Health response to inadequate hygiene measures. ${ }^{8}$

\section{DISCUSSION}

A foodborne illness outbreak among tourists travelling through Sydney was epidemiologically linked to the consumption of contaminated food from a restaurant in Gold Coast, Queensland. The rapid and synchronous onset of severe vomiting and short incubation period was consistent with a toxin-mediated food poisoning. This 
is caused by bacterial toxins produced by $S$. aureus or $B$. cereus and associated with consumption of ready-toeat foods such as cold meats or sushi often contaminated by food handlers who have been colonized by these pathogens. Based on the clinical presentation, testing for SFP was undertaken in addition to routine cultures and antigen testing. However, protocols to detect toxinproducing $B$. cereus from clinical samples (stool or blood) are not routine and hence laboratory testing for $B$. cereus was not undertaken. Vomitus sample would have been the most suitable for testing; however, patients had stopped vomiting on hospital presentation. Detection of SE in stools of two patients combined with isolation of a similar organism from the food handling environment was suggestive epidemiological evidence of SFP.

Staphylococcal food poisoning occurs when food contaminated by colonized food handlers carrying SE in their noses or on their hands that contain enterotoxins produced by $S$. aureus is consumed. ${ }^{9}$ SEs are produced in food stored at elevated temperature $\left(30-37^{\circ} \mathrm{C}\right)$ following contamination with $S$. aureus. ${ }^{2,10}$ Even after the bacteria are destroyed by heat, the potent gastrointestinal exotoxins which are resistant to heat and proteolytic enzymes (particularly SEA) remain active in the digestive tract producing intoxication even at very low inoculums. ${ }^{2,11}$ Investigators were only able to test the rice consumed by the group; however, environmental sampling conducted at the restaurant revealed that 7 of 10 swabs were positive for an enteric pathogen, including three with $S$. aureus. Due to the time lapse of the investigation, food specimens from the majority of foods consumed were not obtained for testing and toxin typing was not possible for environmental samples to make a molecular comparison. That there were also $B$. cereus detected in four environmental samples, suggests inadequate sanitizing of food-handling surfaces, indicative of poor food hygiene practices at the restaurant and potential environmental sources of food contamination. The detection of Salmonella in food also suggested poor food-handling practices at the restaurant.

A limitation of this investigation is the potential for recall bias, although the group had a clearly defined meal schedule with no reported deviation from this schedule. Therefore, recall bias would have been limited. The clinical specimens (stool and blood) were not tested for toxin-producing $B$. cereus which could have also caused the outbreak. The failure to isolate $S$. aureus from one of the stool samples could be due to pre-analytical factors like delay in receipt of the specimen, delay in plating to media, organism burden in the sample, presence of inhibitors of $S$. aureus in stool sample as well as analytical factors like sensitivity of detection of organism by the culture method. Additionally, due to the two-day delay in conducting the environmental investigation, investigators were unable to obtain and test the majority of foods consumed by the group.

Although diagnosis of SFP is mainly clinical, toxin detection aids with epidemiological investigations especially in large and multijurisdictional outbreaks. ${ }^{12}$ Public health officials should consider including SFP in laboratory testing for outbreaks characterized by predominance of vomiting, absence of fever and simultaneous onsets. Where preliminary laboratory tests are negative for bacterial and viral pathogens, consideration should be given to further testing based on the preceding factors. Increased availability of improved diagnostic methods could help with the detection of toxin-mediated foodborne diseases.

\section{Conflicts of interest}

None declared.

\section{Funding}

This outbreak investigation was conducted as part of routine public health work. All authors are employed by the Public Health Service under their respective state governments.

\section{Acknowledgements}

The authors would like to acknowledge all the clinical, laboratory and environmental health staff who conducted the field investigations.

\section{References}

1. Kirk M et al. Foodborne illness, Australia, circa 2000 and circa 2010. Emerging Infectious Diseases, 2014, 20:1857-1864. doi:10.3201/eid2011.131315 pmid:25340705

2. Argudín MÁ, Mendoza MC, Rodicio MR. Food poisoning and Staphylococcus aureus enterotoxins. Toxins, 2010, 2:17511773. doi:10.3390/toxins2071751 pmid:22069659 
3. Lund $\mathrm{M}$ et al. Detection of Campylobacter spp. in chicken fecal samples by real-time PCR. Journal of Clinical Microbiology, 2004 42:5125-5132. doi:10.1128/JCM.42.11.5125-5132.2004 pmid: 15528705

4. Malorny B et al. Diagnostic real-time PCR for detection of Salmonella in food. Applied and Environmental Microbiology, 2004, 70:7046-7052. doi:10.1128/AEM.70.12.70467052.2004 pmid:15574899

5. Vu DT et al. Detection of Shigella by a PCR assay targeting the ipaH gene suggests increased prevalence of shigellosis in Nha Trang, Vietnam. Journal of Clinical Microbiology, 2004, 42:2031-2035. doi:10.1128/JCM.42.5.2031-2035.2004 pmid:15131166

6. Becker K, Roth R, Peters G. Rapid and specific detection of toxigenic Staphylococcus aureus: use of two multiplex PCR enzyme immunoassays for amplification and hybridization of staphylococcal enterotoxin genes, exfoliative toxin genes, and toxic shock syndrome toxin 1 gene. Journal of Clinical Microbiology, 1998, 36:2548-2553. pmid:9705390
7. FSANZ. Guidelines for microbiological examination of readyto-eat foods. Canberra, Food Standards Australia New Zealand (FSANZ), 2001.

8. Foodborne IIIness Outbreak Management Guideline [press release]. Queensland Health, 2013, 15:2006.

9. El-Shenawy $\mathrm{M}$ et al. Cross sectional study of skin carriage and enterotoxigenicity of Staphylococcus aureus among food handlers. Open Journal of Medical Microbiology, 2014.

10. Pillsbury A et al. An outbreak of staphylococcal food poisoning in a commercially catered buffet. Communicable Diseases Intelligence Quarterly Report, 2013, 37:E144-148. pmid:24168088

11. Bergdoll MS. Enterotoxins. Staphylococci and Staphylococcal Infections, 1983, 2:559-598.

12. Asao $T$ et al. An extensive outbreak of staphylococcal food poisoning due to low-fat milk in Japan: estimation of enterotoxin $A$ in the incriminated milk and powdered skim milk. Epidemiology and Infection, 2003, 130:33-40. doi:10.1017/ S0950268802007951 pmid:12613743 